\title{
Dynamic Changes in Endogenous Hormones in Terminal Buds from Different Crown Positions in Sequoia sempervirens (Lamb.) Endl
}

\author{
Shuming Ju \\ Xuzhou Institute of Technology, Xuzhou 221111, China; and Jiangsu \\ Laboratory of Pollution Control and Resource Reuse, Xuzhou Jiangsu \\ 221111, China
}

\section{Lingzhen Ji and Delan Xu \\ Xuzhou Institute of Technology, Xuzhou 221111, China}

Additional index words. abscisic acid, gibberellic acid, indole acetic acid, zeatin-riboside

\begin{abstract}
Endogenous hormones can improve plant resistance and regulate growth and development. To obtain the basis of chemical control technology for improving Sequoia sempervirens resistance in Xuzhou, China, the current study probed the dynamic changes of endogenous hormones in terminal buds from different crown positions in $S$. sempervirens. Enzyme-linked immunosorbent assay (ELISA) was used to measure changes in the contents of endogenous hormones in terminal buds from the upper, middle, and lower lateral branches. The results were as follows: Indole acetic acid (IAA) in all terminal positions had a similar change trend of "rise-drop-rise." Gibberellic acid (GA) in the upper and middle terminal buds showed similar trends of "drop-rise," but GA in the lower lateral branches presented a "rise-drop" trend. Zeatin-riboside (ZR) in all terminal positions had similar change trends of "drop-rise." Abscisic acid (ABA) in all terminal positions had similar change trends of "drop-rise-drop." the trend of (IAA + GA + ZR)/ABA in all terminal positions was the same as that of IAA. Our results confirmed that, in autumn, the high content and ratio of stimulatory endogenous hormones in the terminal bud of $S$. sempervirens induced the terminal bud cells to continue to divide and grow, and the new branches could not be fully lignified and deeply dormant before the onset of low temperatures in winter, which led to a decrease in cold resistance and even the death of the terminal buds.
\end{abstract}

Sequoia sempervirens belongs to cedar, a relict plant. Natural forests of $S$. sempervirens in North America are now found only in a narrow strip along the California coast. $S$. sempervirens, which grows quickly and lives for many years (Chang et al., 2015), is one of the five largest ornamental tree species in the world, and has high value for cultivation and

Received for publication 2 Oct. 2018. Accepted for publication 25 Jan. 2021.

Published online 26 April 2021.

This work was supported by the National Spark Plan Project (no. 2013GA690441), the College Natural Fund of Jiangsu Province (no. 07kjd210198), the Science and Technology Plan Project of Xuzhou (no. XM13B124), and the Plan Project of Xuzhou Institute of Technology (no. xky201013).

S.J., L.J., and D.X. designed the project. S.J. analyzed the data, was responsible for the greenhouse and laboratory experiments, and for corresponding with the editors and reviewers. L.J. revised the manuscript. All authors read and approved the final manuscript.

S.J. is the corresponding author. E-mail: qusm2010@ 163.com.

This is an open access article distributed under the CC BY-NC-ND license (https://creativecommons. org/licenses/by-nc-nd/4.0/). promotion (Ju et al., 2009). Therefore, in past years, $S$. sempervirens has been introduced to various parts of the world. It was first introduced to China in 1972 and has been now cultivated in many southeastern areas (Sheng et al., 2017). S. sempervirens was first planted in Xuzhou on a trial basis in 2000. During the process of cultivation in autumn and winter, its terminal buds were lost and, the next spring, the lateral buds sprouted into new branches. Considering the habitable environment of $S$. sempervirens and the living environment in Xuzhou (Ju et al., 2019; Zhang et al., 2015), the causes of terminal bud death were analyzed. There are three possible explanations. First, terminal bud death in $S$. sempervirens can be the result of low temperatures in winter (Luo et al., 2013). In addition, before the arrival of cold air, the $S$. sempervirens buds are not fully lignified, which contributes to their death. Observation of trees under cultivation has shown that not all terminal buds are dead; the rate of death is $30 \%$. When the degree of lignification is high, a bud does not die. Second, this problem may be a result of a water deficit in $S$. sempervirens. The weather is dry and windy during the winter in Xuzhou, which impairs lignification in $S$. sempervirens branches, so terminal buds die because of a shortage of water (Luo et al., 2013). Observation of trees under cultivation has shown that $S$. sempervirens planted beneath shelter performed better than those planted in the open ground in terms of the death of terminal buds. Third, and last, low temperatures and the shortage of water may combine to cause terminal bud death in S. sempervirens (Zuo et al., 2000).

These options indicate directions for exploring the mechanism of bud death of $S$. sempervirens. However, the death of the terminal bud is a complicated process, and can be influenced by many internal and external factors (Wang et al., 2012). The concentration and ratio of hormones have a significant regulatory effect on plant resistance (Karimi, 2017; Wang et al., 2012; Yuan et al., 2020). Studies have shown that cells resisted freezing temperatures by dehydration or the accumulation of sugar and protein complexes bound to water, thereby avoiding freezing, which destroys cells and leads to death, and all processes are coordinated by hormones and the perception of temperature (Chen et al., 2006; Karimi, 2017). At the same time, most researchers thought that endogenous hormone signaling could adjust the growth and development of buds, and affect woody plant adaptation mechanisms such as bud death (Arora et al., 2003; Pan and Dong, 1998; Wang et al., 2012). During the annual growth cycle of $S$. sempervirens, there are obvious growth rhythms resulting from the influences of the internal environment and external factors that lead to the formation of multiple new branches (Lochard and Schneider, 1981). For plant endogenous hormones, it is generally acknowledged that IAA, GA, and ZR are stimulatory hormones; $\mathrm{ABA}$ is a suppressive hormone. IAA is the most ubiquitous auxin in higher plants, which affects cell division, cell elongation, and cell differentiation (Mwange et al., 2005; Sivaci and Yalcin, 2008). ABA is associated with plant dormancy, meaning that high levels lead to plant dormancy and low levels benefit the plant by releasing dormancy and promoting growth and germination (Sivaci and Yalcin, 2008; Zhang et al., 2020). The hormones GA and ZR not only stimulate the growth of plants, but also are linked to the differentiation of plant buds (Peng et al., 2013; Wu et al., 2017). The regulation of plant endogenous hormones on plant growth and development is related to a certain type of hormone and the ratio between different hormones. Studies have shown that an increased ABA/ GA ratio is closely related to enhanced cold resistance (Zheng et al., 2009).

Numerous studies have explained the physiological mechanisms of endogenous hormones on plant stress resistance, and growth and development (Shi et al., 2006). Currently, no research describes the dynamic changes of endogenous hormones in the terminal buds of $S$. sempervirens. The purpose of our study was 1) to determine the content of endogenous hormones in the terminal buds of S. sempervirens in different seasons; 2) to analyze the variation characteristics of 


\begin{tabular}{lcccccccccccc}
\hline & \multicolumn{10}{c}{ Mo. } \\
\cline { 2 - 13 } Meteorological parameters & 1 & 2 & 3 & 4 & 5 & 6 & 7 & 8 & 9 & 10 & 11 & 12 \\
\hline Mean temperature $\left({ }^{\circ} \mathrm{C}\right)$ & 0.7 & 3.5 & 8.6 & 15.6 & 21.1 & 25.4 & 27.3 & 26.4 & 22.1 & 16.2 & 8.8 & 2.8 \\
Humidity (\%) & 66 & 64 & 62 & 61 & 64 & 67 & 79 & 81 & 75 & 69 & 69 & 66 \\
Total rainfall $(\mathrm{mm})$ & 18 & 20 & 36 & 47 & 66 & 107 & 241 & 133 & 72 & 52 & 27 & 14 \\
\hline
\end{tabular}

endogenous hormones in different seasons, combined with the climatic characteristics of Xuzhou; and 3) to explore the relationship between the content or proportion of endogenous hormones and terminal bud death to provide a theory for the technology of chemical control in the production of $S$. sempervirens in Xuzhou.

\section{Materials and Methods}

Test site location. The test site was located at the Xuzhou Institute of Technology nursery base in Xuzhou, Jiangsu Province, China (lat. $34^{\circ} 15^{\prime} \mathrm{N}$, long. $\left.117^{\circ} 11^{\prime} \mathrm{E}\right)$, which has a warm temperate and semihumid monsoon climate. The annual average temperature is $14.58^{\circ} \mathrm{C}$, the annual average lowest temperature is $-10.52^{\circ} \mathrm{C}(1960-2018)$, and the extreme minimum temperature is $-22.6^{\circ} \mathrm{C}$ (noted in 1969 , which is in our study range of 1960-2018). The annual sunshine duration is 2284 to 2495 $\mathrm{h}$, and the relative sunshine duration is $52 \%$ to $57 \%$. The annual accumulated temperature is $5143.5^{\circ} \mathrm{C}$, the average annual frost-free period is $\approx 210 \mathrm{~d}$, and the average annual precipitation is $853.1 \mathrm{~mm}$. The soil at the test site is a sandy soil, and the $\mathrm{pH}$ is 8.3 (Ju et al., 2020; Wang et al., 2010). Some of the meteorological conditions encountered during the sampling period are presented in Table 1.

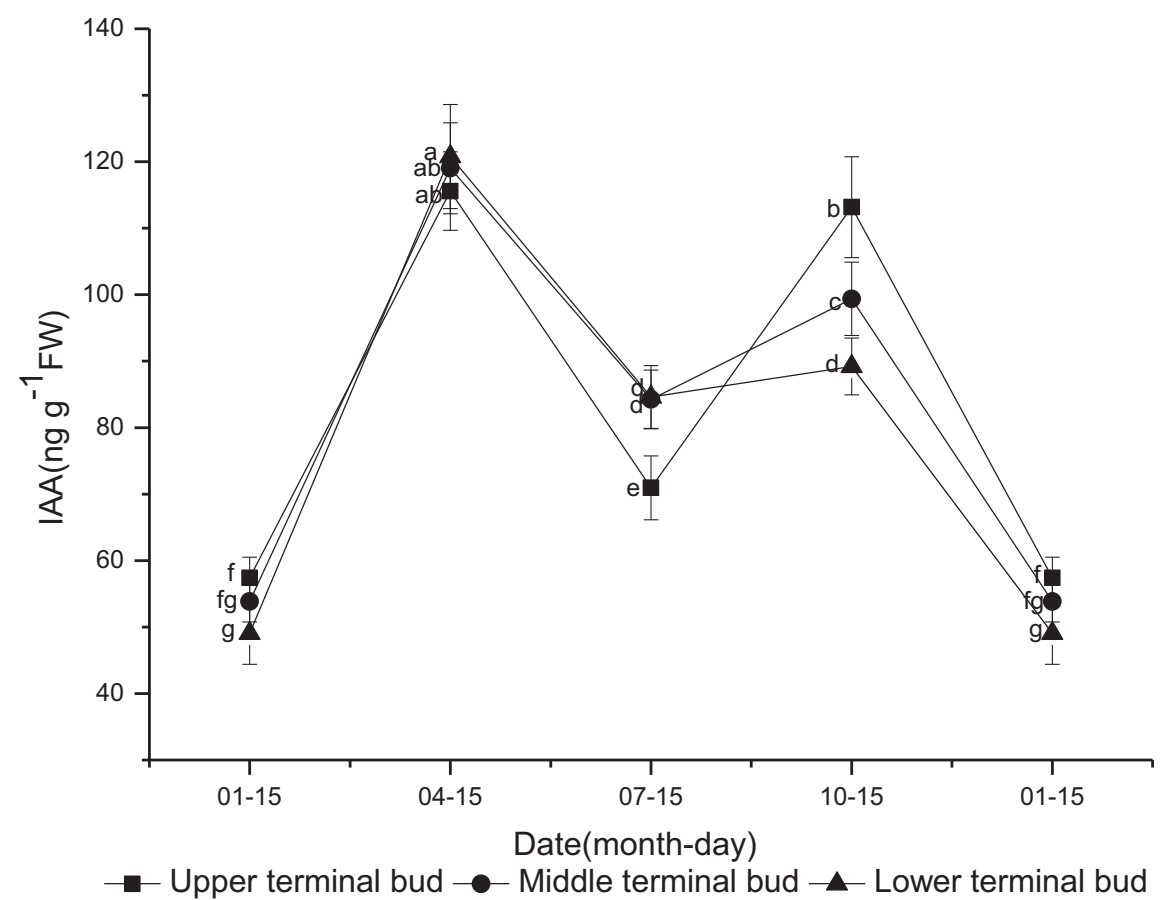

Fig. 1. Change in indole acetic acid (IAA) content in different terminal positions in S. sempervirens. Different letters indicate significant differences at $P<0.05$. FW, fresh weight.
Experiment materials. We used 6-, 8-, and 9-year-old $S$. sempervirens as experiment materials. The sampling times were spring (15 Apr.), summer (15 July), autumn (15 Oct.), and winter (15 Jan.). In case of rain, the sampling time was adjusted by $3 \mathrm{~d}$, so the weather was observed in advance. During sampling, three samples were taken from each plant, and the sampling sites were mainly the upper terminal bud (the terminal bud of the second-layer branch from the top to the bottom of the crown), the middle terminal bud (the terminal bud of the middle-layer branch of the crown), and lower terminal bud (the terminal bud of the bottom-layer branch of the crown). Samples were taken from four directions and each material was equivalent.

Determination of endogenous hormone content. Measurement of the endogenous hormones IAA, GA, ZR, and ABA of $S$. sempervirens terminal buds was performed using ELISA (Wu et al., 1993). The ELISA kit was purchased from China Agricultural University. It measured optical density at $490 \mathrm{~nm}$ and drew a calibration curve, then calculated the IAA, GA, ZR, and ABA levels. Each sample assay was repeated three times and, after excluding abnormal data, the data were averaged for analysis (Zhou et al., 2014). Calculation was performed using the regression method to establish a logit curve.

Data processing. SPSS 19.0 analysis software (SPSS Inc., Chicago, IL) was used to analyze the measurement data. Differences between different treatments were analyzed using one-way analysis of variance with Tukey's test at a significance level of $P<0.05$. Origin 8.0 software (OriginLab Corp., Northampton, MA) was used to produce a plot.

\section{Results}

Changes in IAA content in different terminal positions in $S$. sempervirens. As shown in Fig. 1, the trend of IAA in terminal buds from different crown positions was "rise-drop-rise." In winter, IAA content in terminal buds was low, and plants were in a period of dormancy. In spring, IAA content was generally high, which indicates active bud growth. In summer, IAA content at the different positions was decreasing, which indicates that bud growth had declined. However, IAA content increased again, showing that the plants had an additional growing season in autumn. In addition, at that time, IAA content of the upper terminal bud was greater than that of the middle and lower terminal buds.

Changes in GA content in different terminal positions in $S$. sempervirens. As shown in Fig. 2, the trends of GA in the upper, middle, and lower terminal buds were obviously different. In winter, the GA content in the upper and middle terminal buds were greater than those of the lower buds. From winter to summer, GA content in the upper and middle terminal buds decreased gradually, whereas those of the lower terminal buds increased gradually. From summer to autumn, GA content in the upper and middle terminal buds increased gradually; however, those of the lower terminal buds decreased gradually. In autumn, GA content of the upper terminal buds were the greatest; those of the lower terminal buds were the lowest.

Changes in ZR content in different terminal positions in $S$. sempervirens. As shown in Fig. 3, changes in ZR content in the upper, middle, and lower terminal buds resembled a "V" shape, or a "drop-rise" trend. In winter, ZR content in the middle and lower terminal buds was generally high. From winter to summer, ZR content in the upper, middle, and lower terminal buds increased gradually, reaching its lowest point of the year when $S$. sempervirens is dormant in summer. From summer to autumn, ZR content from the different crown positions increased gradually, and not much difference was observed among the three locations. The basic biological function of ZR is to promote cell division. Because ZR content in terminal buds at different positions was not low in autumn, $S$. sempervirens was still growing quickly in autumn. 


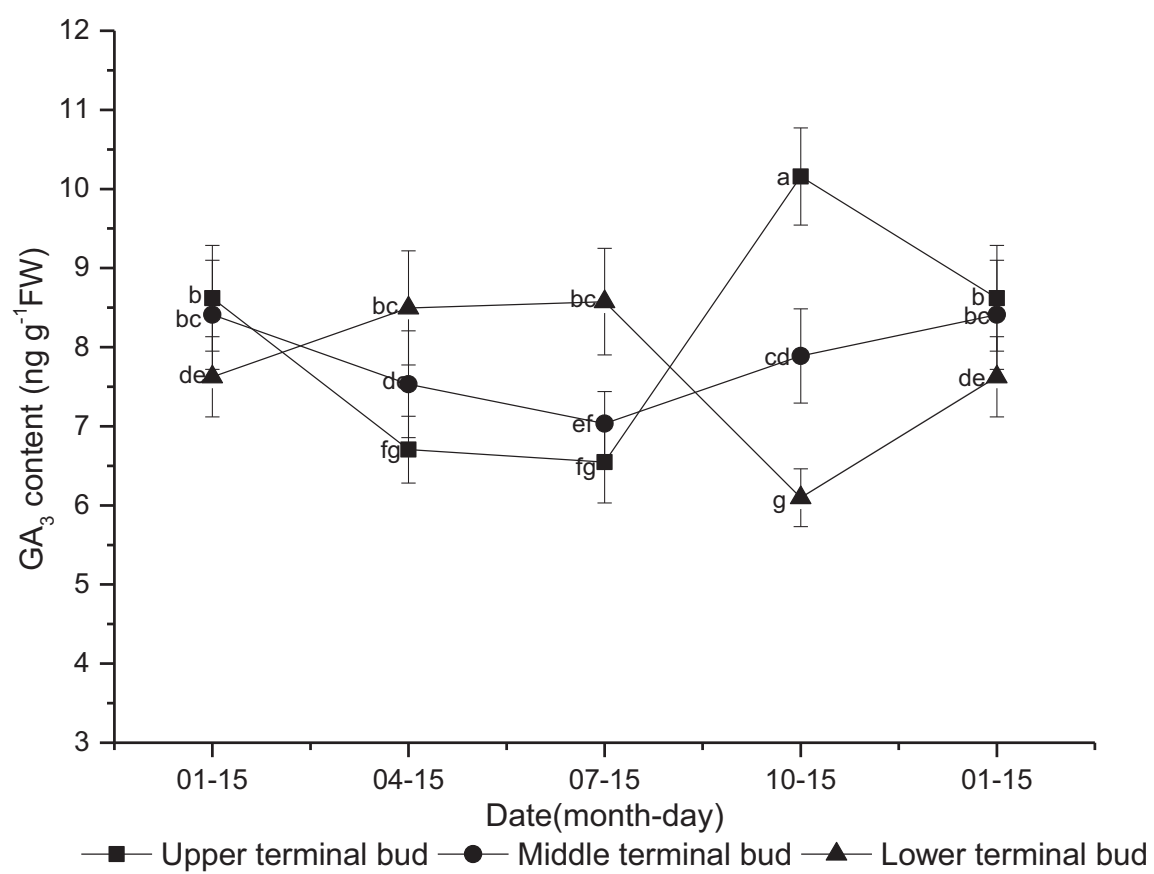

Fig. 2. Change in gibberellic acid $\left(\mathrm{GA}_{3}\right)$ content in different terminal positions in S. sempervirens. Different letters indicate significant differences at $P<0.05 . \mathrm{FW}$, fresh weight.

Changes in ABA content in different terminal positions in S. sempervirens. As shown in Fig. 4, the trend of ABA content in terminal buds from different crown positions was "drop-rise-drop." In winter, when the plants were dormant, ABA content in the upper terminal bud was the lowest, and those in the middle and lower terminal buds were generally greater. From winter to spring, ABA content in terminal buds from different crown positions decreased significantly, reaching

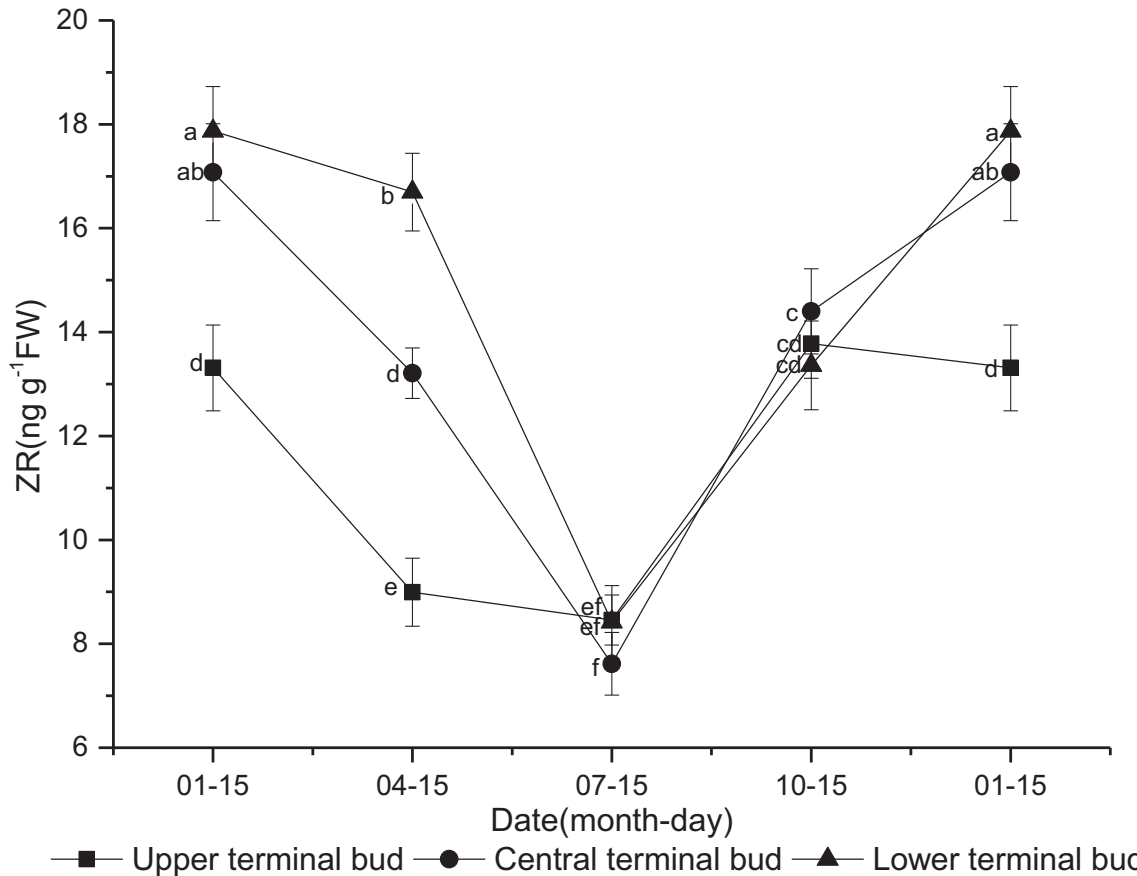

Fig. 3. Change in zeatin-riboside (ZR) content in different terminal positions in S. sempervirens. Different letters indicate significant differences at $P<0.05$. FW, fresh weight.
Changes in the ratio $(I A A+G A+Z R) /$ $A B A$ in different terminal positions in $S$. sempervirens. As shown in Fig. 5, the trend in the ratio of $(\mathrm{IAA}+\mathrm{GA}+\mathrm{ZR}) / \mathrm{ABA}$ in terminal buds from different crown positions in $S$. sempervirens was similar to that of IAA. From winter to spring and from summer to autumn, the ratios increased, and they reached a peak in spring. From spring to summer and from autumn to winter, the ratios of the upper, middle, and lower terminal buds were all decreasing, and they reached a minimum. In winter and summer, the ratios were all less than one, indicating that inhibitory hormones dominated and the plant was dormant. However, in spring and autumn, the ratios were more than one, which shows that growth-promoting hormones dominated and the plant was actively growing.

\section{Discussion}

Ecological factors such as temperature, drought, photoperiod, and so on affect endogenous hormones levels (Sivaci and Yalcin, 2008; Zhang et al., 2020). Plant physiology studies have shown that the effect of external factors on plant growth and development is realized through the regulation mechanism of endogenous hormones (Pan and Dong, 1998). Our study shows that endogenous hormones levels vary with the seasons (Figs. 1-5). After the introduction of $S$. sempervirens to Xuzhou, IAA content in terminal buds from different crown positions showed a similar change trend of "rise-drop-rise" (Fig. 1), which was consistent with the growth type of a double peak in spring and autumn. Some studies have shown that IAA content in scales of Lilium longiflorum (Okubo et al., 1988), leaves of Cinnamomum pauciflorum, and terminal buds of Paulownia (Wang et al., 2012) also demonstrate a similar seasonal variation trend. Considering the climate characteristics of the introduced region (Xuzhou, East-central China; Table 1) and provenance region (Eureka, western North American) (Zhang et al., 2015), the growth patterns of a double peak were influenced by climate and regulated by IAA (Bai and $\mathrm{Yu}, 2005)$. In the cool autumn in Xuzhou, a rapid growth of $S$. sempervirens led to new branches being unable to become sufficiently lignified and deeply dormant before the onset of low temperatures in winter, which may reduce cold resistance and be one of the mechanisms of bud death in winter. Rajagopal et al. (1988) showed that a low auxin (1-naphthyl acetic acid) content promoted lignification of Cuscuta. Hang and Wang (1982) suggested that for the strongly cold-resistant varieties of Malus sp., the lignification of the branches was greater in degree and occurred at a faster rate.

GA has the effects of promoting high growth, enhancing apical dominance, and delaying senescence (Yan et al., 2010). Studies of the seasonal dynamic change of GA content in plants such as Actinidia arguta (Yuan et al., 2020) and Lilium longiflorum (Sivaci and Yalcin, 2008) showed that low-temperature stress in winter 


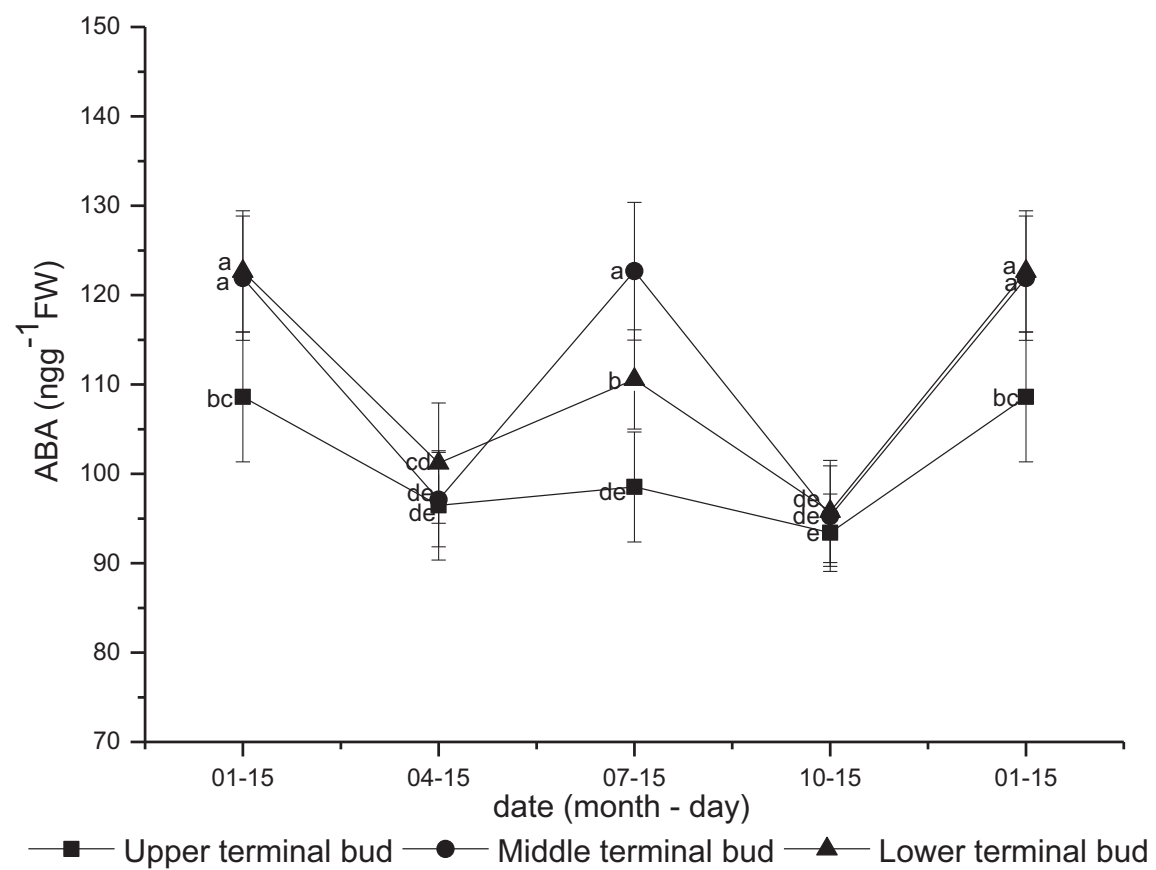

Fig. 4. Change in abscisic acid (ABA) content in different terminal positions in S. sempervirens. Different letters indicate significant differences at $P<0.05$. FW, fresh weight.

reduced GA content, which induced dormancy, inhibited plant growth, and was conducive to safe overwintering of plants. However, our study showed inconsistent results. In the upper and middle terminal buds, GA content in winter was significantly greater than that in spring and summer, which is not conductive to $S$. sempervirens hibernation and safe overwintering. S. sempervirens could have inherited

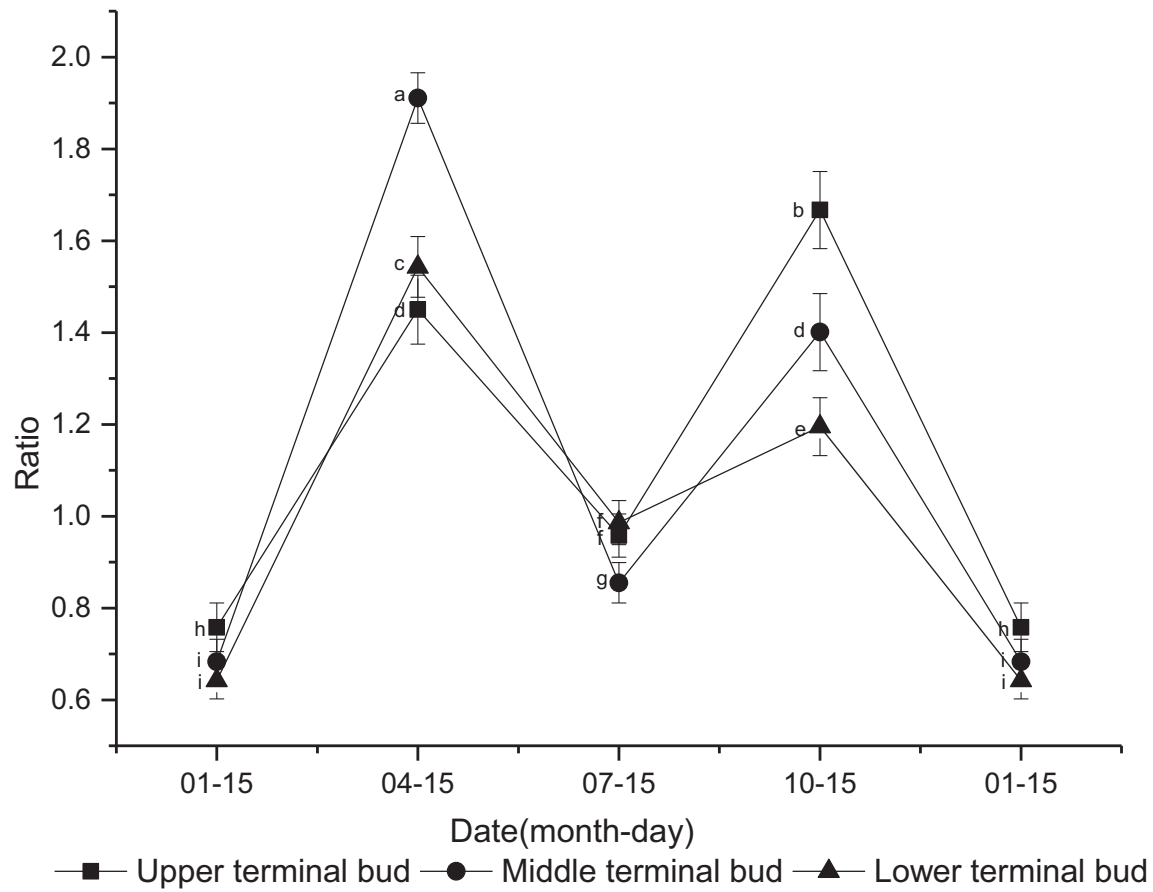

Fig. 5. Change in the ratio [(indole acetic acid + gibberellic acid + zeatin-riboside)/abscisic acid] in different terminal positions in $S$. sempervirens. Different letters indicate significant differences at $P<0.05$ lower terminal bud reached its lowest point of the year, but GA content of the upper and middle terminal buds was very high, which shows that $S$. sempervirens continued to grow by rapid elongation in autumn. This situation was unfavorable for the formation of a dormant bud. Patel and Franklin (2009) believed that reducing GA concentration could limit plant elongation and thus improve plant cold resistance.

The basic physiological function of ZR is to promote cell division (Wang et al., 2012). Studies by Lisabeth (1971) on Populus tremula, Acer platanoides, and Betula verrucose; by Kong et al. (2009) on Pseudotsuga menziesii; and by Pan et al. (2000) on Camellia sinensis showed that ZR content in buds increased obviously at the bud initiation stage. Studies on the dynamic change of ZR content showed that low-temperature stress reduced ZR content in plants in winter, as seen in Ginkgo biloba, Salix babylonica, Acer mono, and Cotinus coggygria (Zhang et al., 2020), and Lilium longiflorum (Sivaci and Yalcin, 2008), which reduced cell division of terminal buds, induced plant dormancy, and was beneficial to safe overwintering. However, our study showed inconsistent results. ZR content in winter was significantly greater than that in spring, summer, and autumn. S. sempervirens could have inherited from its ancestors this growth and germinate characteristic that acts during the winter wet season in western North America during December and January (Zhang et al., 2015). A high concentration of $Z R$ in winter was not conducive to hibernation and safe overwintering of $S$. sempervirens.

ABA is a well-known stress hormone (Yang et al., 2008; Zheng et al., 2009). Studies have shown that ABA plays an important role in balancing growth and climate adaptation (Yu et al., 2019). In our study, ABA content in terminal buds of $S$. sempervirens showed a trend of "drop-rise-drop-rise." High temperatures in summer and low temperatures in winter induced an increase in ABA content in buds, which improved resistance to temperature stress. Previous studies showed that high temperatures in summer can increase ABA content significantly in the terminal buds of Paulownia (Wang et al., 2012), and low temperatures in summer can increase ABA content significantly in the buds of Acer saccharum (Dumbroff et al., 1979). In our study, ABA content from different terminal bud positions reached its lowest value of the year in autumn, which shows that the plants grew well during this period and were not prepared for dormancy.

The effects of endogenous hormones on plant growth and development depend not only on the absolute contents of some endogenous hormones, but also on the dominant hormone during a certain period, which assumes a guiding role (Brenner, 1989). The ratio of (IAA + $\mathrm{GA}+\mathrm{ZR}$ )/ABA could reflect plant growth conditions. A low ratio indicates that suppressive hormones are dominant and plants are dormant; a high ratio indicates that stimulatory hormones are dominant and plants are actively growing (Li et al., 2007; Wang et al., 2012). In 
our study, on the whole, the range of change from winter to spring was greater than that from summer to autumn. From summer to autumn, the ratio of (IAA $+\mathrm{GA}+\mathrm{ZR}) / \mathrm{ABA}$ increased. According to the previous singlehormone analysis, this was mainly a result of a greater IAA, GA, and ZR content, rather than a decrease in ABA content in autumn. The larger ratio of (IAA + GA + ZR)/ABA indicates that $S$. sempervirens was still in the active growth period in autumn, which is also consistent with the peak growth of $S$. sempervirens in the autumn. This made the branches unable to be sufficiently lignified and deeply dormant before the onset of low temperatures in winter, which might be one of the mechanisms of bud death in winter. Practices have also proved this point in reverse. The application of the growth inhibitor paclobutrazol and calcine during autumn could inhibit significantly the growth of redwood branches and improve the cold resistance of the tree (Ju et al., 2019, 2020).

The regulation of endogenous hormones in plant morphology is a complicated process. One kind of hormone can participate in multiple physiological processes (Culter and Vlitos, 1962). Our study showed that the death of terminal buds of $S$. sempervirens was closely related to endogenous hormones, and confirmed that, in autumn, the high content and ratio of stimulatory endogenous hormones in the terminal bud of $S$. sempervirens induced the terminal bud cells to continue to divide and grow. This did not allow the new branches to become fully lignified and deeply dormant before the onset of low temperatures in winter, which led to a decrease in cold resistance and even the death of the terminal buds.

\section{Literature Cited}

Arora, R., L.J. Rowland, and K. Tanino. 2003. Induction and release of bud dormancy in woody perennials: A science comes of age. HortScience 38:911-921, doi: 10.21273/HORTSCI.38.5.911.

Bai, B. and T. Yu. 2005. Changes in endogenous phytohormone content of larch seedlings during terminal bud growth. For. Sci. Technol. 4:8-10. <http:// d.wanfangdata.com.cn/periodical/lykj200504003>.

Brenner, M.L. 1989. Hormonal control of assimilate partitioning regulation in the sink. Acta Hort. 239: 141-148.

Chang, X.Y., B.C. Xu, K.Y. Zhang, Q.S. Yu, and Y.Q. Wang. 2015. Research progress and development suggestion of Sequoia sempervirens in our country. Modern Agr. Sci. Technol. 12:180-186. <http://d. wanfangdata.com.cn/periodical/ahny201512113>.

Chen, J., K.W. Pan, and B. Gu. 2006. Physiological function and mechanism of abscisic acid in plants under stress. Plant Physiol. J. 42(6):1176-1182.

Culter, H.G. and A.J. Vlitos. 1962. The natural auxins of the sugar cane. Physiol. Plant. 15(1):27-42, doi: 10.1111/1399-3054.ep15754438.

Dumbroff, E.B., D.B. Cohen, and D.P. Webb. 1979. Seasonal levels of abscisic acid in buds and stems of Acer saccharum. Physiol. Plant. 45: 211-214, doi: 10.1111/1399-3054.ep13278160.

Hang, Y. and Z. Wang. 1982. Cytological determinations of cold resistance of fruit trees (Malus genue). Acta Hort. Sinica 9(8):23-30.

Ju, S.M., M.X. Gao, and D.L. Xu. 2009. Study on the asexual rapid propagation of cold-resistant Sequoia sempervirens. Pract. For. Technol. 1: 23-25. <https://www.cnki.net/kcms/doi/10.134 56/j.cnki.lykt.2009.01.012>.
Ju, S.M., D.L. Xu, C.Y. Zhang, L.Z. Ji, T.C. Yin, Z.L. Li, and Z.Q. Lu. 2019. Influence of paclobutrazol on the growth and photosynthesis of Sequoia sempervirens seedings. J. Hort. Res. 27(1): 21-30, doi: 10.2478/johr-2019-0003.

Ju, S.M., D.L. Xu, C.Y. Zhang, J.Q. Lu, X.Q. Jiang, and L.Z. Ji. 2020. Induction of tolerance by chlorocholine chloride in Sequoia sempervirens seedlings under natural cooling and drought. J. For. Sci. 66 (6):236-243, doi: 10.17221/118/2019-JFS.

Karimi, R. 2017. Potassium-induced freezing tolerance is associated with endogenous abscisic acid, polyamines and soluble sugars changes in grapevine. Scientia Hort. 215:184-194, doi: 10.1016/j.scienta.2016.12.018.

Kong, L.S., S.R. Abrams, S.J. Owen, A.V. Niejenhuis, and P.V. Aderkas. 2009. Dynamic changes in concentrations of auxin, cytokinin, ABA and selected metabolites in multiple genotypes of douglas-fir (Pseudotsuga menziesii) during a growing season. Tree Physiol. 29(2):183-190, doi: 10.1093/treephys/tpn009.

Li, X.M., X.Y. He, and W. Chen. 2007. Effects of elevated atmospheric $\mathrm{CO}_{2}$ concentration on endogenous hormones in Gingko leaves. Chinese J. Appl. Ecol. 18(7):1420-1424. <http://www. cjae.net/CN/Y2007/V18/I07/1420>.

Lisabeth, E. 1971. Cytokinins in buds and leaves during growth, maturity and aging (with a comparison of two bio-assays). Biochem. Physiol. Pflanz. 162:547-558, doi: 10.1016/S00153796(17)31188-5.

Lochard, R.G. and G.W. Schneider. 1981. Stock and scion growth relationships and the dwarfing mechanism in apple. Hort. Rev. 3:315-375, doi: 10.1002/9781118060766.ch7.

Luo, J.X., H.W. Wu, Y.J. Gu, J. Gao, and H.L. Wang. 2013. A preliminary report on introduction and acclimation of Sequoia sempervirens in Miyi of Sichuan. J. Sichuan For. Sci. Technol. 34(2):40-42. <https://www.cnki.net/kcms/ doi/10.16779/j.cnki.1003-5508.2013.02.008>.

Mwange, K., H. Hou, Y. Wang, X. He, and K. Cui. 2005. Opposite patterns in the annual distribution and time-course of endogenous abscisic acid and indole-3-acetic acid in relation to the periodicity of cambial activity in Eucommia ulmoides Oliv. J. Expt. Bot. 56(413):1017-1028, doi: $10.1093 / \mathrm{j} x \mathrm{~b} / \mathrm{eri095}$.

Okubo H., Chijiwa, M., and S. Uemoto. 1988. Seasonal changes in leaf emergence from scale bulbiets during scaling and endogenous plant hormone levels in easter lily (Lilium longiflorum Thunb.). J. Fac. Agr. Kyushu Univ. 33(1.2):9-15. Pan, R.Z. and Y.D. Dong. 1998. Phytophysiology. Higher Education Press, Beijing, China.

Pan, G.S., S.R. Shen, L.S. Qian, and B.Q. Wu. 2000. Endogenous hormone level and its regulation mechanism during growth of tea plant shoot: (I) Changes of hormone content in the growth of tea plant shoot. Chaye 26(3):139-143, doi: 10.3969/ J.ISSN.0577-8921.2000.03.007.

Patel, D. and K.A. Franklin. 2009. Temperature-regulation of plant architecture. Plant Signal. Behav. 4(7):577-579, doi: 10.4161/psb.4.7.8849.

Peng, L., L. Zhou, and Y. Wang. 2013. Dynamic changes in zeatin nucleoside (ZR) content of mango cutback fruit branch. J. Southern Agr. 44(1):39-42, doi: 10.3969/j:issn.2095-1191.2013.1.39.

Rajagopal, I., S. Ramachandiran, and S. Mahadevan. 1990. Plant Growth Substances 1988. SpringerVerlag, Berlin, Heidelberg.

Sheng, W.X., J.R. Cai, M. Gao, and J.C. Jiang. 2017. A study on the characteristics of stand growth of Sequoia sempervirens plantation. South China For. Sci. 45(1):10-13. <https:// www.cnki.net $/ \mathrm{kcms} / \mathrm{doi} / 10.16259 / \mathrm{j}$.cnki.361342/s.2017.01.003>.
Shi, C.J., Y. Liu, and T. Jing. 2006. Review on stress-resistance of phytohormone. World For. Res. 19(5):21-26, doi: 10.3969/j.issn.10014241.2006.05.004.

Sivaci, A. and L. Yalcin. 2008. The seasonal changes in endogenous levels of indole-3-acetic acid, gibberellic acid, zeatin and abscisic acid in stems of some apple varieties (Malus sylvestris Miller). Asian J. Plant Sci. 7(3):319-322, doi: 10.3923/ajps.2008.319.322.

Wang, J., H. Jia, Y.N. Wang, and M.Y. Peng. 2010. Temperature variation characteristics analysis in Xuzhou during 1960-2008. J. Xuzhou Normal Univ. (Natural Science Edition) 28(3):70-74, doi: 10.3969/j.issn.1007-6573.2010.03.017.

Wang, Y.M., Z. Liu, and X.F. Niu. 2012. Dynamic change of endogenous hormones in terminal buds from different crown position of one-year-old Paulownia. Scientia Silvae Sinicae 48(7):61-65. <http:// d.wanfangdata.com.cn/periodical/lykx201207010>.

Wu, Q., J.C. Han, and Z.P. He. 1993. Dynamic changes of the endogenous hormones during refrigeration. J. China Agr. Univ. 4:11-14.

Wu, T., W.H. Liang, B.S. Shi, and S.J. Xu. 2017. Effects of temperature and gibberellin on seed germination of carmine flower. J. Hebei For. Sci. Technol. 1:15-18. <http://d.wanfangdata. com.cn/periodical/heblykj201701004>.

Yan, S.P., R.H. Yang, L.F. Guan, and Q.Y. Wang. 2010. A comparative on the plant endogenous hormones of transgenic and non-transgenic hybrid aspen tissue-cultured plantlets. Scientia Silvae Sinicae 46(9):40-44. <http://d.wanfangdata. com.cn/periodical/lykx201009007>.

Yang, R.L., Y.C. Wang, and Y.X. Chang. 2008. Morphogenesis and changes in endogenous phytohormones in Reaumuria trigyna Maxim during fissurate growth. J. Plant Ecol. 32(2):385-391, doi: 10.3773/j.issn.1005-264x.2008.02.016.

Yu, D., H. Wildhagen, S. Tylewicz, P.C. Miskolczi, R.P. Bhalerao, and A. Polle. 2019. Abscisic acid signalling mediates biomass trade-off and allocation in poplar. New Phytol. 223:1192-1203, doi: 10.1111/nph.15878.

Yuan, Y., Z.G. Dai, B.X. Zhang, T.H. Wang, Z. Zhang, T. Jiang, and M.Y. Yu. 2020. Changes in tissue structure and endogenous hormones of Actinidia arguta branches during over-wintering period. Acta Bot. Boreal. Occident. Sin. 40(2):279-286. < http://d.wanfangdata.com.cn/ periodical/xbzwxb202002012>.

Zhang, S., J. Dai, and Q. Ge. 2020. Responses of autumn phenology to climate change and the correlations of plant hormone regulation. Sci. Rep. 10: 9039, doi: 10.1038/s41598-020-65704-8.

Zhang, J.W., A. D'Rozario, J.M. Adams, Y. Li, X.Q. Liang, F.M. Jacques, T. Su, and Z.K. Zhou. 2015. Sequoia maguanensis, a new Miocene relative of the coast redwood, Sequoia sempervirens, from China: Implications for paleogeography and paleoclimate. Amer. J. Bot. 102:103-118, doi: 10.3732/ajb.1400347.

Zheng, G.S., S.P. Gai, and W.L. Gai. 2009. Changes of endogenous hormones during dormancy release by chilling in tree peony. Scientia Silvae Sinicae 45(2):48-52. <http://d.wanfangdata.com.cn/perio dical/lykx200902009>.

Zhou, P.A., D.Y. Liu, D. Zong, and H. Wu, Y. Zheng, and Z.C. He. 2014. Dynamic changes of endogenous hormones in lateral buds of Populus yunnanensis during different seasons. For. Res. 27(1): 113-119. < https://www.cnki.net/kcms/doi/10.1327 5/j.cnki.lykxyj.2014.01.019>.

Zuo, X.D., R.P. Qi, and J.P. Shao. 2000. Introduction and ecological adaptability of Sequoia sempervirens Endl. in China. Yunnan For. Sci. Technol. 4 36-40. < https://www.cnki.net/kcms/doi/10.16473/ j.cnki.xblykx1972.2000.04.006>. 